\title{
Capabilities of the CMS detector for studies of hard probes in heavy ion collisions at the LHC
}

\section{Igor Lokhtin* (for the CMS Collaboration)}

D.V. Skobeltsyn Institute of Nuclear Physics, M.V. Lomonosov Moscow State University, Moscow, Russia

E-mail: Igor.Lokhtin@cern.ch

\begin{abstract}
The capabilities of the CMS experiment to study properties of hot and dense QCD-matter created in heavy ion collisions at the CERN Large Hadron Collider with the perturbative processes (socalled "hard probes") are presented. Detailed studies from complete simulations of the CMS detectors in $\mathrm{Pb}+\mathrm{Pb}$ collisions at $\sqrt{s}=5.5 \mathrm{TeV}$ per nucleon pair are presented in view of two hard probes: quarkonium and $\gamma$-jet production.
\end{abstract}

High-pT Physics at LHC - Tokaj'08

March 16 - 192008

Tokaj, Hungary

${ }^{*}$ Speaker. 


\section{Introduction}

The study of the fundamental theory of the strong interaction (Quantum Chromodynamics, QCD) in new, unexplored extreme regimes of super-high densities and temperatures is one of the primary goals of the modern high energy physics. The experimental and phenomenological study of multiple particle production in ultrarelativistic heavy ion collisions is expected to provide valuable information on the (thermo)dynamical behaviour of QCD matter in the form of a quark-gluon plasma (QGP), as predicted by lattice QCD calculation. A detailed description of the potential of CMS to carry out a series of representative $\mathrm{Pb}-\mathrm{Pb}$ measurements has been presented in [1].

Heavy ion observables accessible to measurement with CMS include:

- "Soft" probes [2]: global particle and energy rapidity densities, elliptic flow and spectra of low transverse momentum hadrons. These observables are mostly sensitive to the space-time evolution of the system once thermalization has set in and thus carry information about the thermodynamical properties of the produced QCD matter.

- "Hard" probes: quarkonia, heavy quarks, jets, $\gamma$-jet and high- $p_{T}$ hadrons, which are produced with high transverse momenta $p_{T}$ or large masses $M$ (much greater than the typical QCD scale of confinement: $p_{T}, M \gg \Lambda_{\mathrm{QCD}}=200 \mathrm{MeV}$ ). The hard probes production cross sections can be described in the framework of perturbative QCD theory. Such hard particles are produced in the very early stages of the evolution of the system and thus are potentially affected by final-state interactions as they traverse the produced medium. Modifications with respect to the "vacuum QCD" spectra and cross-sections measured in proton-proton collisions, provide direct information on the dynamical and transport properties of the system: initial parton densities, transport coefficient of the medium, critical energy density.

In this contribution, the detailed study for a complete simulation of the CMS detectors in $\mathrm{Pb}+\mathrm{Pb}$ collisions at $\sqrt{s}=5.5 \mathrm{TeV}$ per nucleon pair are presented in view of two hard probes: quarkonium and $\gamma$-jet production. The results of similar studies for high- $p_{T}$ hadrons are presented during this Workshop in another talk [3].

\section{CMS detector}

CMS is a general purpose experiment at the LHC designed to explore the physics at the $\mathrm{TeV}$ energy scale [4]. Since the CMS detector subsystems have been designed with a resolution and granularity adapted to cope with the extremely high luminosities expected in the proton-proton running mode, CMS can also deal with the large particle multiplicities anticipated for heavy-ion collisions. A detailed description of the detector elements can be found in the corresponding Technical Design Reports [5, 6, 7, 8]. The central element of CMS is the magnet, a $13 \mathrm{~m}$ long, $6 \mathrm{~m}$ diameter, high-field solenoid (a uniform $4 \mathrm{~T}$ field) with an internal radius of $\approx 3 \mathrm{~m}$.

The tracker covers the pseudorapidity region $|\eta|<2.5$ and is composed of two different types of detectors: silicon pixels and silicon strips. The pixel detector consists of three barrel layers located at 4, 7, $11 \mathrm{~cm}$ from the beam axis with granularity $150 \times 150 \mu \mathrm{m}^{2}$ and two forward layers with granularity $150 \times 300 \mu \mathrm{m}^{2}$ located at the distances of 34 and $43 \mathrm{~cm}$ in z-direction from the 
center of detector. Silicon strip detectors are divided into inner and outer sections and fill the tracker area from $20 \mathrm{~cm}$ to $110 \mathrm{~cm}$ (10 layers) in the transverse direction and up to $260 \mathrm{~cm}$ (12 layers) in longitudinal direction.

The hadronic (HCAL) and electromagnetic (ECAL) calorimeters are located inside the coil (except the forward calorimeter) and cover (including the forward calorimeter) from -5.2 to 5.2 in pseudorapidity. The HF calorimeter covers the region $3<|\eta|<5.2$.

The CMS muon stations cover the pseudorapidity region $|\eta|<2.4$ and consist of drift tube chambers (DT) in the barrel region (MB), $|\eta|<1.2$, cathode strip chambers (CSCs) in the endcap regions (ME), $0.9<|\eta|<2.4$, and resistive plate chambers (RPCs) in both barrel and endcaps, for $|\eta|<2.1$. The RPC detector is dedicated to triggering, while the DT and CSC detectors, used for precise momentum measurements, also have the capability to self-trigger up to $|\eta|<2.1$.
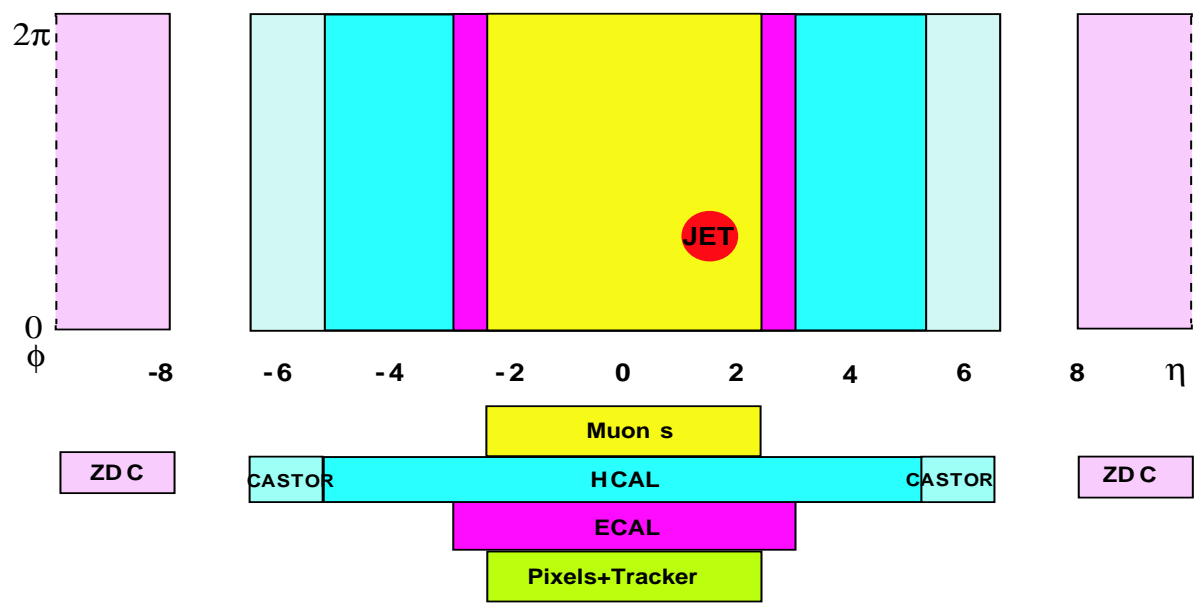

Figure 1: CMS coverage for tracking, calorimetry, and muon identifi cation in pseudo-rapidity $(\eta)$ and azimuth $(\phi)$. The size of a jet with cone $R=0.5$ is also depicted for comparison.

Note that CMS is the largest acceptance detector at the LHC (Fig. 1) with unique detection capabilities also in the very forward hemisphere with the CASTOR $(5.1<|\eta|<6.6)$ and the ZeroDegree (ZDCs, $\left|\eta_{\text {neut }}\right|>8.3$ ) calorimeters [9].

Another key aspect of the CMS hard probe capabilities for heavy ion physics is its unparalleled high-level-trigger (HLT) system running on a filter farm with an equivalent of $\mathscr{O}\left(10^{4}\right) 1.8 \mathrm{GHz}$ CPU units, yielding few tens of Tflops [10]. The HLT system is powerful enough to run "offline" algorithms on every single $\mathrm{Pb}+\mathrm{Pb}$ event delivered by the level-1 trigger, and select the interesting events while reducing the data stream from an average $3 \mathrm{kHz} \mathrm{L1}$ input/output event rate down to $10-100 \mathrm{~Hz}$ written to permanent storage. The resulting enhanced statistical reach for hard probes is a factor of $\times 20$ to $\times 300$ larger, depending on the signal, than for the min-bias (MB) trigger.

\section{Simulation and analysis of $\mathbf{J} / \psi$ and $\Upsilon$ production}

One of the important hard probes at the LHC will be the production of heavy-quark bound states, which should be suppressed in QGP due to colour screening [11]. An intriguing phenomenon is the "anomalously" strong suppression of the $\mathrm{J} / \psi$-meson yields, observed in $\mathrm{Pb}+\mathrm{Pb}$ 
collisions at SPS $[12,13]$. Although the interpretation of this phenomenon as a result of the formation of a QGP is quite plausible, alternative explanations have also been put forward, such as rescattering on co-moving hadrons. The surprisingly similar amount of $\mathrm{J} / \psi$ suppression observed at SPS and RHIC energies [14] is not yet fully understood. Further information on the nature of quarkonia suppression in hot and dense QCD-matter will come from the results at the LHC at the much higher temperatures accessible. CMS features the best dimuon mass resolution of any LHC detector, leading to a clean separation of the various quarkonia states and an improved signal over background ratio. This fact will open up a unique opportunity to study the threshold dissociation behaviour of the whole bottomonium family $\left(\Upsilon, \Upsilon^{*}, \Upsilon^{\prime \prime}\right)$ together with the charmonium one. Since various quarkonium states are predicted to melt at different medium temperatures, scan of corresponding suppression factors will serve as an effective QCD-matter "thermometer".

Event generator HIJING [15] with full GEANT4-based simulation of the tracking of secondaries and simulated detector response were used in the analysis presented. The $J / \psi$ and $\Upsilon$ acceptances on CMS are shown as a function of $p_{T}$ in Fig. 2 for two $\eta$ ranges: full detector $(|\eta|<2.4)$ and central barrel $(|\eta|<0.8)$. Because of their relatively small mass, low momentum $\mathrm{J} / \psi$ 's $(p<4$ $\mathrm{GeV} / c$ ) are mostly not accepted: their decay muons do not have enough energy to traverse the calorimeters and coil, and are absorbed before reaching the muon chambers. The $\mathrm{J} / \psi$ acceptance increases with $p_{T}$, flattening out at $\sim 15 \%$ for $p_{T}>12 \mathrm{GeV} / c$. The $\Upsilon$ acceptance starts at $\sim 40 \%$ at $p_{T}=0 \mathrm{GeV} / c$ and remains constant at $\sim 15 \%$ (full detector) or $5 \%$ (barrel only) for $p_{T}>4 \mathrm{GeV} / c$. The $p_{T}$-integrated acceptance is about $1.2 \%$ for the $\mathrm{J} / \psi$ and $26 \%$ for the $\Upsilon$, assuming the input theoretical distributions.

In the central barrel of the CMS detector, the dimuon reconstruction efficiency remains above $80 \%$ for all multiplicities whereas the purity decreases slightly with increasing multiplicities $d N_{c h} / d \eta$ but also stays above $80 \%$ even at as high as $d N_{c h} /\left.d \eta\right|_{\eta=0}=6500$. If (at least) one of the muons is detected in the endcaps, the efficiency and purity drop due to stronger reconstruction cuts. Nevertheless, for the $d N_{c h} /\left.d \eta\right|_{\eta=0} \approx 2000$ multiplicity realistically expected in central $\mathrm{Pb}+\mathrm{Pb}$ at $\mathrm{LHC}$, the efficiency (purity) remains above 65\% (90\%) even including the endcaps.

At the $\Upsilon$ mass, the dimuon mass resolution for muon pairs in the central barrel, $|\eta|<0.8$, is $54 \mathrm{MeV} / c^{2}$. In the full pseudorapidity range, the dimuon mass resolution is about $1 \%$ of the quarkonium mass: $35 \mathrm{MeV} / c^{2}$ at the $\mathrm{J} / \psi$ mass and $86 \mathrm{MeV} / c^{2}$ at the $\Upsilon$ mass. There is a slight dependence of the mass resolution on the event multiplicity. Increasing the multiplicity from $d N / d \eta=0$ to 2500 degrades the mass resolution of the reconstructed $\Upsilon$ from 86 to $90 \mathrm{MeV} / \mathrm{c}^{2}$.

Fig. 3 shows the opposite-sign dimuon mass distributions, for the high and low multiplicity cases and full acceptance $(|\eta|<2.4)$. The different quarkonia resonances appear on top of a continuum due to the various sources of decay muons: $\pi+K$, charm and bottom decays. Assuming that the CMS trigger and acceptance conditions treat opposite-sign and like-sign muon pairs equally, the combinatorial like-sign background can be subtracted from the opposite-sign dimuon mass distribution, giving us a better access to the quarkonia decay signals. The statistics of $J / \psi$ and $\Upsilon, \Upsilon^{\prime}$ and $\Upsilon^{\prime \prime}$ with both muons in $|\eta|<2.4$ region expected in one month of data taking are 140000, 20000, 5900 and 3500 correspondingly for the multiplicity $d N_{c h} /\left.d \eta\right|_{\eta=0}=5000$ and 180000, 25000, 7300, 4400 for the multiplicity $d N_{c h} /\left.d \eta\right|_{\eta=0}=2500$. The signal-to-background ratios are $0.6,0.07$ for $\mathrm{J} / \psi$ and $\Upsilon$ 's for $d N_{c h} /\left.d \eta\right|_{\eta=0}=5000$ correspondingly and $1.2,0.12$ for $d N_{c h} /\left.d \eta\right|_{\eta=0}=2500$. The signal-to-background ratio (the number of events) collected in one month for the dimuons in $\mathrm{J} / \psi$ 

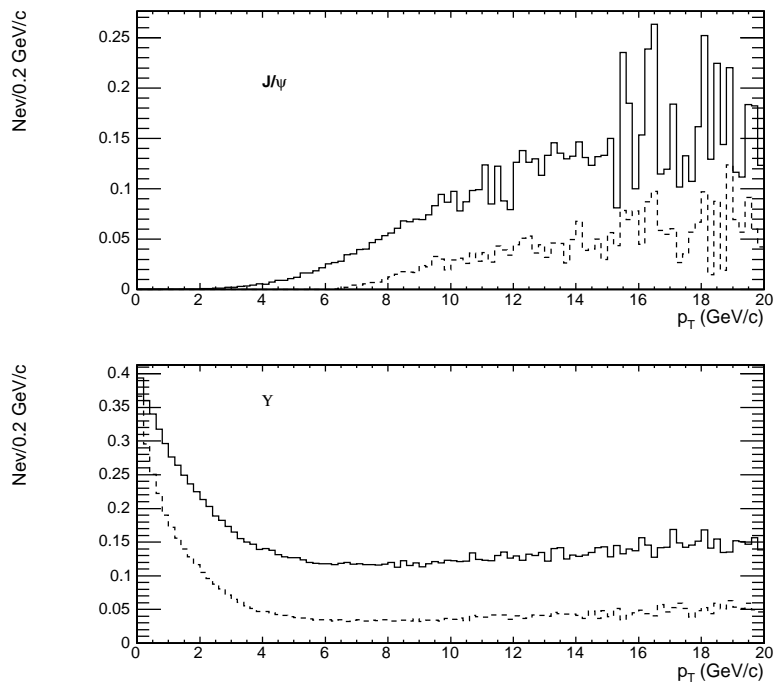

Figure 2: $\mathrm{J} / \psi$ (top) and $\Upsilon$ (bottom) CMS acceptances (convoluted with trigger effi ciencies) as a function of $p_{T}$, in the full detector $(|\eta|<2.4$, solid line) and only in the central barrel $(|\eta|<0.8$, dashed line).
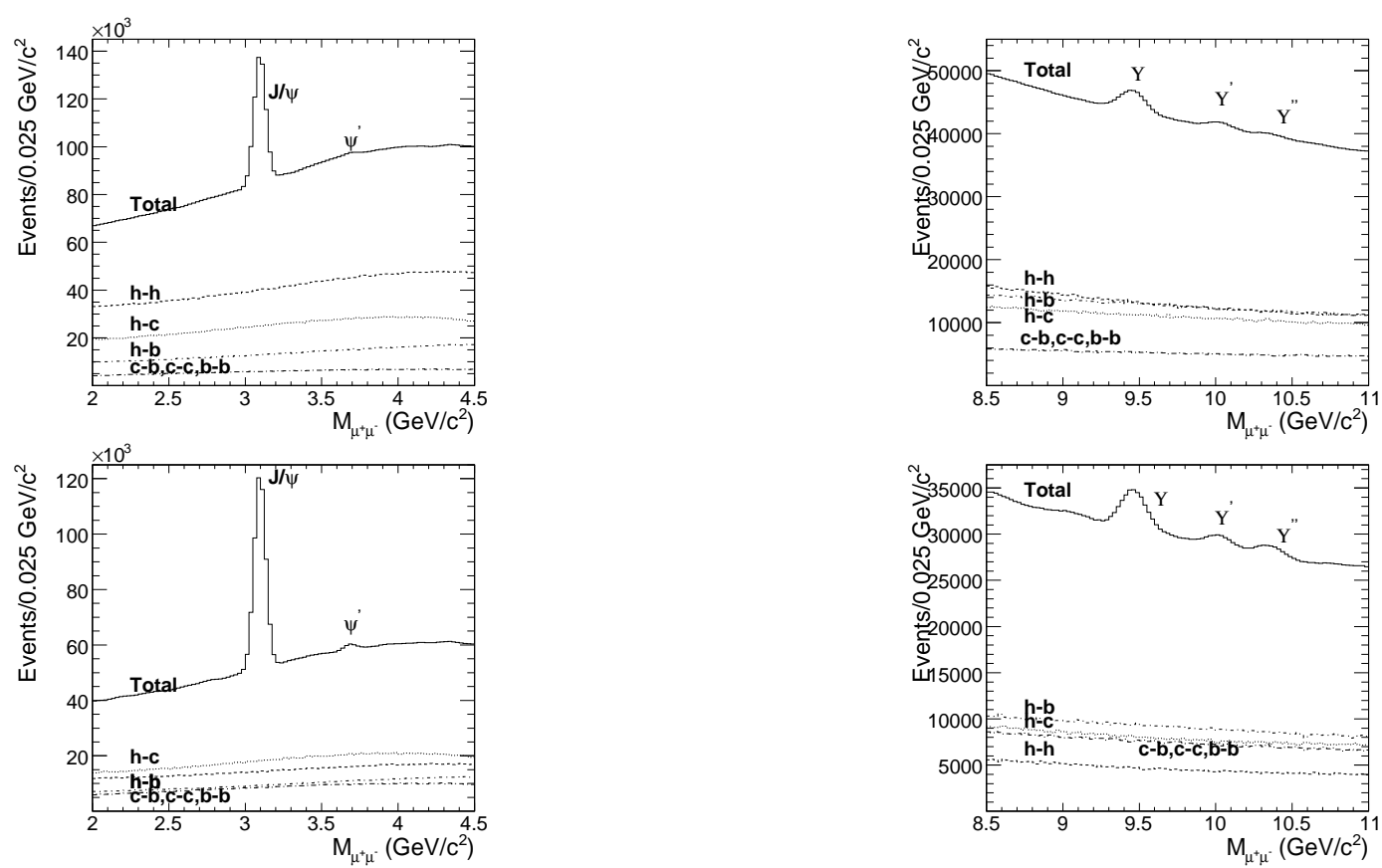

Figure 3: Dimuon mass distributions within $|\eta|<2.4$ for $\mathrm{Pb}+\mathrm{Pb}$ events with $d N_{c h} /\left.d \eta\right|_{\eta=0}=5000$ (top) and $d N_{c h} /\left.d \eta\right|_{\eta=0}=2500$ (bottom) in the $\mathrm{J} / \psi$ (left) and $\Upsilon$ (right) mass regions. The main background contributions are also shown: $h, c$ and $b$ stand for $\pi+K$, charm, and bottom decay muons, respectively.

and $\Upsilon$ mass regions with both particles in $|\eta|<0.8$ region are 2.75 (12600) and 0.52 (6000) for $d N_{c h} /\left.d \eta\right|_{\eta=0}=5000$ and 4.5 (11600), 0.97 (6400) for $d N_{c h} /\left.d \eta\right|_{\eta=0}=2500$. The background and reconstructed resonance numbers are in a mass interval $\pm \sigma$, where $\sigma$ is the mass resolution. 
These quantities have been calculated for an integrated luminosity of $0.5 \mathrm{nb}^{-1}$ assuming an average luminosity $\mathscr{L}=4 \times 10^{26} \mathrm{~cm}^{-2} \mathrm{~s}^{-1}$ and a machine efficiency of $50 \%$. The expected statistics are large enough to allow further offline analysis for example in correlation with the centrality of the collision or the transverse momentum of the resonance.

\section{Photon-tagged jet production}

Another important hard probe very sensitive to the initial conditions of the produced QCDmatter in heavy-ion collisions, is the QCD jet production. It is expected that final state in-medium interactions should reduce the energy of the jet partons ("jet quenching") and result in mediummodified jet fragmentation [16]. Recent RHIC data on high- $\mathrm{p}_{T}$ hadron production [17] are consistent with jet quenching predictions. However full event-by-event reconstruction of jets in heavy ion collisions is rather complicated in the lower energy RHIC experiments. In CMS, large transverse momentum probes can be isolated experimentally from the soft particle background of the collision. In particular, full jet reconstruction and high- $\mathrm{p}_{T}$ particle reconstruction in the high multiplicity environment of a $\mathrm{Pb}+\mathrm{Pb}$ collision are possible [1]. At the $\mathrm{LHC}$, the production rates for jet pairs with transverse energy $E_{T}>50 \mathrm{GeV}$ are several orders of magnitude larger than at RHIC. Thus, high statistics systematic studies will be possible in a controlled perturbative regime, far beyond the limits of RHIC. The one of the important jet-related observables accessible to study in heavy ion collisions at CMS will be the $\gamma$-jet (and $Z$-jet) channel provides a very clean means to determine medium-modified parton fragmentation functions (FFs) [18]. Since the prompt $\gamma$ is not affected by final-state interactions, its transverse energy $\left(E_{T}^{\gamma}\right)$ can be used as a proxy of the away-side parton energy $\left(E_{T}^{\mathrm{jet}} \approx E_{T}^{\gamma}\right.$ ) before any jet quenching has taken place in the medium. The FF, i.e. the distribution of hadron momenta, $1 / N_{\text {jets }} d N / d z$, relative to that of the parent parton $E_{T}^{\text {jet }}$, can be constructed using $z=p_{T} / E_{T}^{\gamma}$ or, similarly, $\xi=-\ln z=\ln \left(E_{T}^{\gamma} / p_{T}\right)$, for all particles with momentum $p_{T}$ associated with the jet.

Full CMS simulation-reconstruction studies of the $\gamma$-jet channel have been carried out [19], where the isolated $\gamma$ is identified in $\operatorname{ECAL}\left(R_{i s o l}=0.5\right)$, the away-side jet axis $\left(\Delta \phi_{\gamma-j e t}>3 \mathrm{rad}\right)$ is reconstructed in ECAL+HCAL, and the momenta of hadrons around the jet-axis $\left(R_{\text {jet }}<0.5\right)$ are measured in the tracker. The event generators PYTHIA [20] (non-quenched partons) and PYQUEN [21] (quenched partons) were used to simulate signal $\gamma+$ jet events, and HYDJET [21] was used to model the underlying (background) heavy ion event (also without or with jet quenching). For this study, the $10 \%$ most central $\mathrm{Pb}+\mathrm{Pb}$ collisions were selected by the impact parameter of the lead nuclei, yielding an average mid-pseudorapidity density of about 2400 (2200) charged particles in the quenched (unquenched) HYDJET background. In total, $4000 \gamma$-jet events in the CMS acceptance for $E_{T}^{\gamma}>70 \mathrm{GeV}$ and $\left|\eta^{\gamma}\right|<2$ and about 40000 (125000) QCD background events for the quenched (unquenched) case are simulated. This corresponds to the expected yields for one running year of $\mathrm{Pb}+\mathrm{Pb}$ data taking with an integrated luminosity of $0.5 \mathrm{nb}^{-1}$. The working point for this analysis was set to $60 \%$ signal efficiency, leading to a background rejection of about $96.5 \%$, and to a signal-to-background ratio of 4.5 for $0-10 \%$ central quenched $\mathrm{Pb}+\mathrm{Pb}$.

The obtained FFs for photon-jet events with $E_{T}^{\gamma}>70 \mathrm{GeV}$ - after subtraction of the underlyingevent tracks using a $\mathrm{R}=0.5$ cone transverse to the jet - are shown in Fig. 4 for central quenched $\mathrm{Pb}+\mathrm{Pb}$ collisions. Medium modified FFs are measurable with high significance (the systematic 

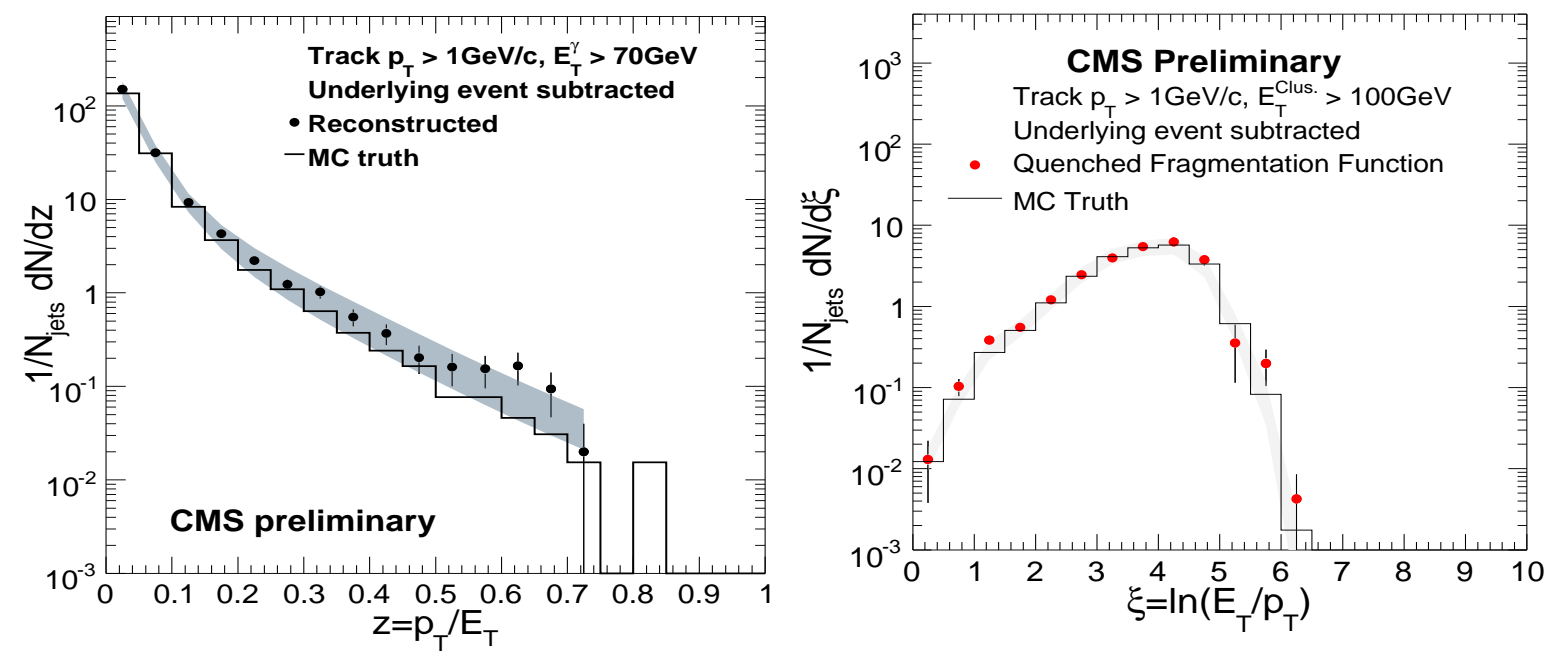

Figure 4: Generated (histogram) and reconstructed (points) fragmentation functions as a function of $z$ (left) and $\xi$ (right) for quenched partons. Statistical errors correspond to an integrated luminosity of $0.5 \mathrm{nb}^{-1}$. The estimated systematic error is represented as the shaded band.

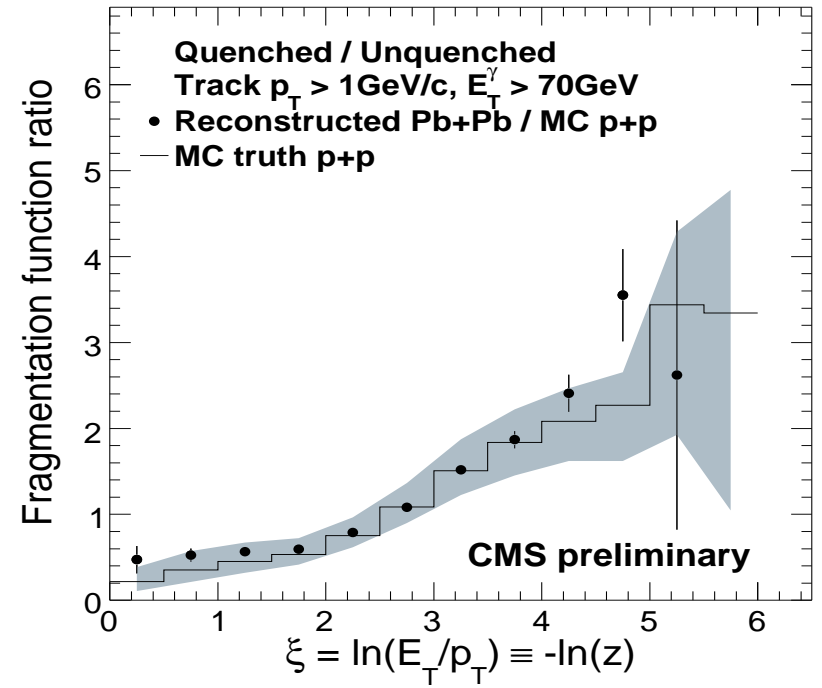

Figure 5: Generated (histogram) and reconstructed (points) ratio of quenched (PYQUEN) and unquenched (PYTHIA) fragmentation functions as a function of $\xi$.

uncertainties being dominated by the low jet reconstruction efficiency for $E_{T}^{\text {jet }}=30-70 \mathrm{GeV}$ ) in the ranges $z<0.7$ or $0.2<\xi<5$. The overall capability to measure the medium-induced modification of jet fragmentation functions in the $\gamma+$ jet channel can be illustrated by comparing the fully reconstructed quenched fragmentation function to the unquenched MC truth distribution (Fig. 5). 


\section{Summary}

With its large acceptance, nearly hermetic fine granularity hadronic and electromagnetic calorimetry, and good muon and tracking systems, CMS is an excellent device for the study of hard probes (such as quarkonia, jets, photons and high- $\mathrm{p}_{T}$ hadrons) in heavy ion collisions at the LHC.

\section{Acknowledgments}

The author wishes to express the gratitude to the members of CMS Collaboration for providing the materials and the organizers of the Workshop "High-pT Physics at LHC" for the warm welcome and the hospitality. The author also gratefully acknowledge support from Russian Foundation for Basic Research (grants No 08-02-91001 and No 08-02-92496) and Grants of President of Russian Federation (No 1007.2008.2 and No 1456.2008.2).

\section{References}

[1] D. d'Enterria (ed.) et al. (CMS Collaboration), J. Phys. G 34 (2007) 2307.

[2] F. Sikler (CMS Collaboration), in these Proceedings.

[3] K. Krajczar (CMS Collaboration), in these Proceedings.

[4] A. de Roeck (ed.) et al.(CMS Collaboration), J. Phys. G 34 (2007) 995.

[5] CMS HCAL Technical Design Report, CERN/LHCC 97-31, 1997.

[6] CMS MUON Technical Design Report, CERN/LHCC 97-32, 1997.

[7] CMS ECAL Technical Design Report, CERN/LHCC 97-33, 1997.

[8] CMS Tracker Technical Design Report, CERN/LHCC 98-6, 1998.

[9] M. Albrow et al. (CMS/TOTEM Collaborations), CERN-LHCC-2006-039/G-124, 2006.

[10] G. Roland et al. (CMS Collaboration), J. Phys. G 34 (2007) S733.

[11] T. Matsui and H. Satz, Phys. Lett. B 178 (1986) 416.

[12] B. Alessandro et al. (NA50 Collaboration), Eur. Phys. J. C 39 (2005) 335.

[13] B. Alessandro et al. (NA50 Collaboration), Eur. Phys. J. C 49 (2007) 559.

[14] A. Adare et al. (PHENIX Collaboration), Phys. Rev. Lett. 98 (2007) 232301.

[15] M. Gyulassy and X.-N. Wang, Comput. Phys. Commun. 83 (1994) 307.

[16] R. Baier, D. Schiff and B.G. Zakharov, Annual Rev. Nucl. Part. Sci. 50 (2000) 37.

[17] RHIC White Papers, Nucl. Phys. A 757 (2005) 28.

[18] F. Arleo et al. JHEP 0411 (2004) 009.

[19] C. Loizides (CMS Collaboration), arXiv:0804.3679.

[20] T. Sjostrand, S. Mrenna and P. Skands, JHEP 0605 (2006) 026.

[21] I.P. Lokhtin and A.M. Snigirev, Eur. Phys. J. C 45 (2006) 211. 